\title{
Thermal cyclotron and annihilation radiation in strong magnetic fields
}

\author{
V.G. BEZCHASTNOV
}

Ioffe Institute of Physics and Technology, 194021, St.Petersburg, Russia

A.D. KAMINKER

Ioffe Institute of Physics and Technology, 194021, St.Petersburg, Russia

\begin{abstract}
The cyclotron and the one-photon annihilation emissions are investigated for a strongly magnetized thermal electron-positron plasmas. The annihilation spectral component is significant when the particle number density $N$ exceeds some critical value, $N_{c r}(T, B)$. For $T \sim 10^{8}-10^{9} \mathrm{~K}$ and $B \sim 10^{12}-10^{13} \mathrm{G}$, this condition can be fulfilled at $N<10^{22} \mathrm{~cm}^{-3}$, which is realistic for neutron star magnetospheres.
\end{abstract}

\subsection{Introduction}

The $e^{-} e^{+}$-plasma in strong magnetic fields of neutron stars can be thought to be responsible for $\mathrm{X}$-ray and $\gamma$-ray radiation of radio pulsars and $\gamma$-ray bursters. In the emitting regions of these objects, the cyclotron emission and one-photon pair annihilation can be important. Separately, they have been investigated by many authors (see, e.g., Bezchastnov and Pavlov 1991, Harding 1986, 1991, and the references therein). However the comparison of these mechanisms has not been performed even for the simplest case of thermal plasmas. We consider the total emission spectra and find the domain of temperatures $T$ and magnetic fields $B$ where the annihilation component is significant for realistic particle number densities $N<10^{22} \mathrm{~cm}^{-3}$. 


\subsection{Spectra of radiation}

Quantum cyclotron emission and one-photon pair annihilation are characterized by the emissivities (summed over polarizations) $j_{c}$ and $j_{a}$, respectively. For the photon propagation across $\vec{B}$ in a tenuous nondegenerate thermal plasma, we obtain

$$
\begin{aligned}
& \frac{j_{c}}{N_{-}+N_{+}}=\frac{2 \alpha b \omega}{A} \mathrm{e}^{-\frac{\omega}{2 t}} \sum_{\nu=\nu_{c}}^{\infty} \mathrm{e}^{-\frac{\nu b}{\omega t}} \sum_{n=0}^{n_{\max }} \frac{\left|D_{n+\nu, n}\right|}{\sqrt{\left(\omega_{c}^{2}-\omega^{2}\right)\left(\omega_{a}^{2}-\omega^{2}\right)}} \\
& \frac{j_{a}}{N_{-} N_{+}}=\frac{4 \pi^{3} \alpha b \lambda_{c}^{3} \omega}{A^{2}} \mathrm{e}^{-\frac{\omega}{t}} \sum_{\nu=0}^{\nu_{a}} \sum_{n=0}^{n_{\max }} \frac{\left(2-\delta_{n, 0}\right)\left|D_{n+\nu, n}\right|}{\sqrt{\left(\omega_{c}^{2}-\omega^{2}\right)\left(\omega_{a}^{2}-\omega^{2}\right)}}
\end{aligned}
$$

Here $\alpha=e^{2} / \hbar c, \lambda_{c}=\hbar / m c, \omega$ is photon energy in units of $m c^{2}, A$ normalizes the thermal distribution function, $b=B / B_{c r}\left(B_{c r}=m^{2} c^{3} / e \hbar=\right.$ $\left.4.41 \times 10^{13} \mathrm{G}\right), t=T / m c^{2}, N_{\mp}$ denote the $e^{\mp}$ number density,

$$
\begin{aligned}
& \omega_{c, a}(\nu, n)=\sqrt{1+2(n+\nu) b} \mp \sqrt{1+2 n b}, \\
& D_{n^{\prime}, n}=b\left(n+n^{\prime}\right)\left[F_{1}^{2}+F_{2}^{2}-F_{3}^{2}-F_{4}^{2}\right]-F_{3}^{2}-F_{4}^{2},
\end{aligned}
$$

$F_{1}=F_{n^{\prime}-1, n}, F_{2}=F_{n^{\prime}, n-1}, F_{3}=F_{n^{\prime}-1, n-1}, F_{4}=F_{n^{\prime}, n}$ are normalized Laguerre functions of the argument $\omega^{2} / 2 b$, and

$$
\nu_{c, a}(\omega)=\frac{\omega}{b}\left(\frac{\omega}{2} \pm 1\right), \quad n_{\text {max }}(\omega, \nu)=\frac{1}{2 b}\left[\left(\frac{\nu b}{\omega}-\frac{\omega}{2}\right)^{2}-1\right] .
$$

The emissivity $j=j_{c}+j_{a}$ is shown in Figs. 1-3. The cyclotron features are produced in radiative transitions $|n+\nu\rangle \rightarrow|n\rangle+|\omega\rangle$ of the particles at $\omega<\omega_{c}(\nu, n)$, while the annihilation ones are caused by the reactions $|n+\nu\rangle+|n\rangle \rightarrow|\omega\rangle$ between $e^{-}$and $e^{+}$at $\omega>\omega_{a}(\nu, n) \geq \omega_{a}(0,0)=2$.

At $\omega \gg b \gg t$, one may keep the only terms $n=0$ in Eq. (1) and replace the sum over $\nu$ by the integral. Then

$$
\begin{aligned}
\frac{\left\langle j_{c}\right\rangle}{N_{-}+N_{+}}= & \frac{\alpha(1+\omega)}{2 \pi^{3 / 2} \sqrt{b}}\left\{\left(1+\frac{2}{\omega}\right)\left[1+\frac{t \omega}{b} \ln \left(1+\frac{2}{\omega}\right)\right]\right\}^{-1 / 2} \times \\
& \exp \left\{-\frac{2}{b}-\frac{\omega}{t}+\frac{\omega^{2}}{2 b}\left(1+\frac{2}{\omega}\right)\left[\frac{2}{\omega}-\ln \left(1+\frac{2}{\omega}\right)\right]\right\} .
\end{aligned}
$$

These spectra are in good agreement with the exact spectra (1). Averaging Eq. (2) over energy interval $2 \leq \omega \leq 1+\sqrt{1+2 b}$ between the first and the second annihilation peaks we obtain

$$
\frac{\left\langle j_{a}\right\rangle}{N_{-} N_{+}}=2 \sqrt{\pi} \alpha \lambda_{c}^{3} b^{-2} t^{-1 / 2} \exp \left(-\frac{2}{b}\right)
$$




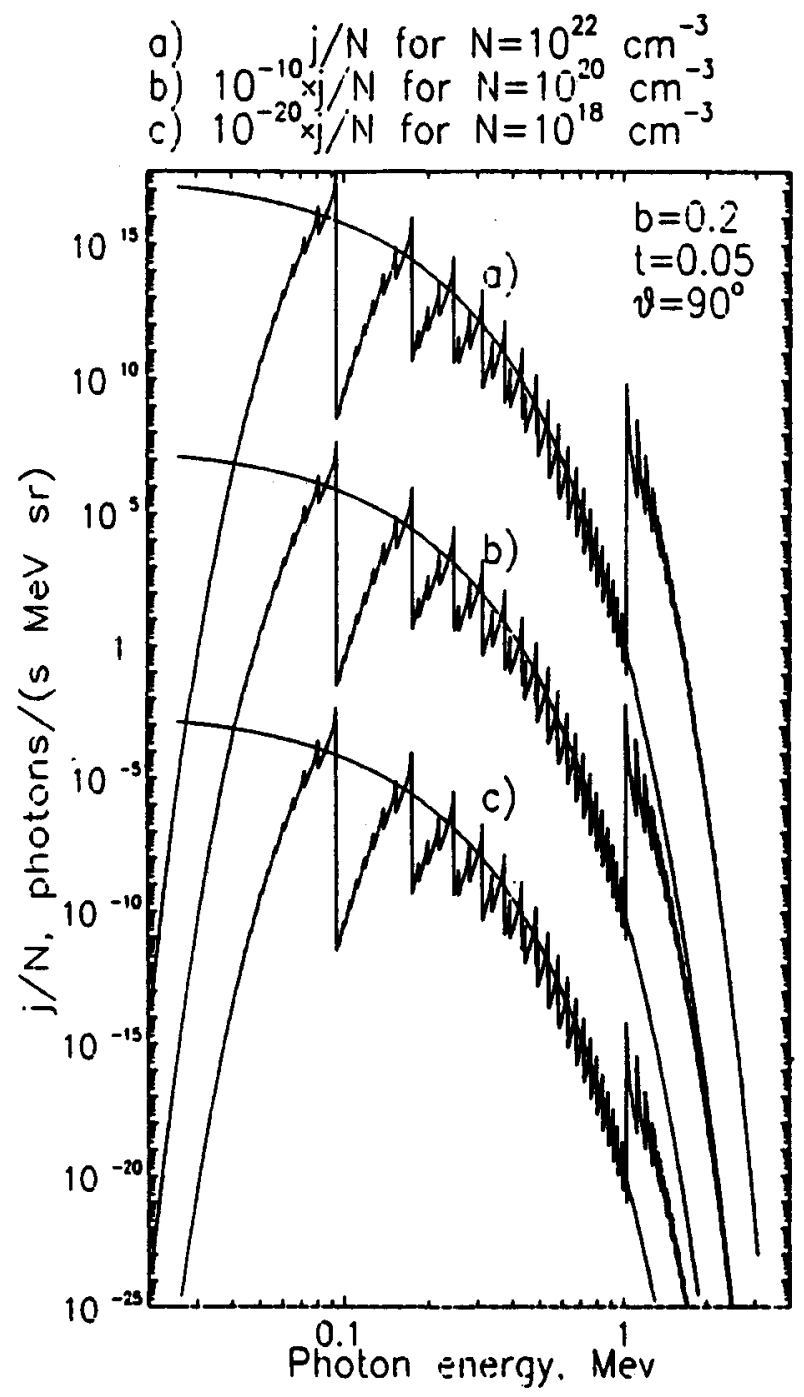

Fig. 30.1 Spectra of differential emissivity $j / N$ as a sum of the cyclotron and one-photon pair annihilation radiations in $e^{-e^{+}}-$plasma with $T=$ $0.05 m c^{2}$ and $B=0.2 B_{c . r}$ for different number densities $N_{-}=N_{+}=N$. Smooth solid lines show the averaged cyclotron spectra (6).

The ratio of Eq. (7) to Eq. (6) at $\omega=2$ gives the critical number density,

$$
N_{c r}=\frac{3 \sqrt{2}}{4 \pi^{2}} \lambda_{c}^{-3} b^{3 / 2} \sqrt{t}\left(1+\frac{1.39 t}{b}\right)^{-1 / 2} \exp \left(\frac{1.23}{b}-\frac{2}{t}\right) \text {. }
$$

For $N>N_{c r}$, the annihilation spectral component forms a jump at the main threshold $(E=1.022 \mathrm{Mev}$ ) followed by a sequence of the fine structure peaks (Fig. 3). The annihilation features are more pronounced on the cyclotron background, when the condition $N>N_{c r}$ is stronger. $N_{c r}(T, B)$ shows sharp growth with increasing $T$ and/or with decreasing $B$ (Fig. 4). 
Bezchastnov \& Kaminker: Thermal cyclotron and annihilation radiation

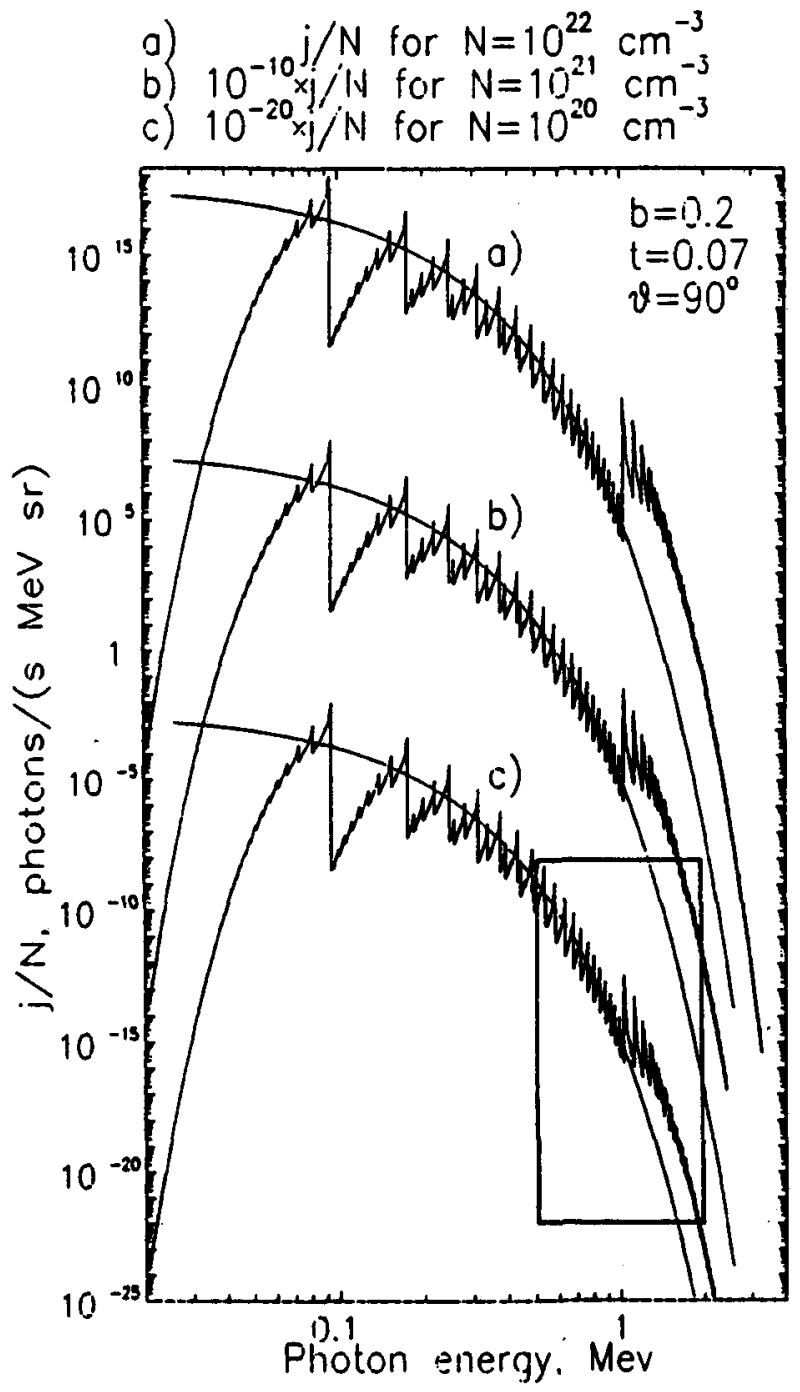

Fig. 30.2 Same as in Fig. 1 for $T=0.07 m c^{2}$. The spectrum inside the rectangle is presented separately in Fig. 3.

\subsection{Discussion}

Soft thermal-like spectra have been observed in the X-ray pulsar state of the 5 March 1979 event, and in the soft $\gamma$-ray repeaters (Mazets et al., 1982, Golenetskii et al., 1984). The fits yield $T \sim(3-5) \times 10^{8} \mathrm{~K}$. Thus one can expect to observe the one-photon annihilation features in the spectra of these soft sources under quite realistic conditions $\left(B>10^{12} \mathrm{G}, N<10^{22} \mathrm{~cm}^{-3}\right)$.

The work was partly supported by the ESO C\&EE Grant A-01-068. 
Bezchastnov \& Kaminker: Thermal cyclotron and annihilation radiation

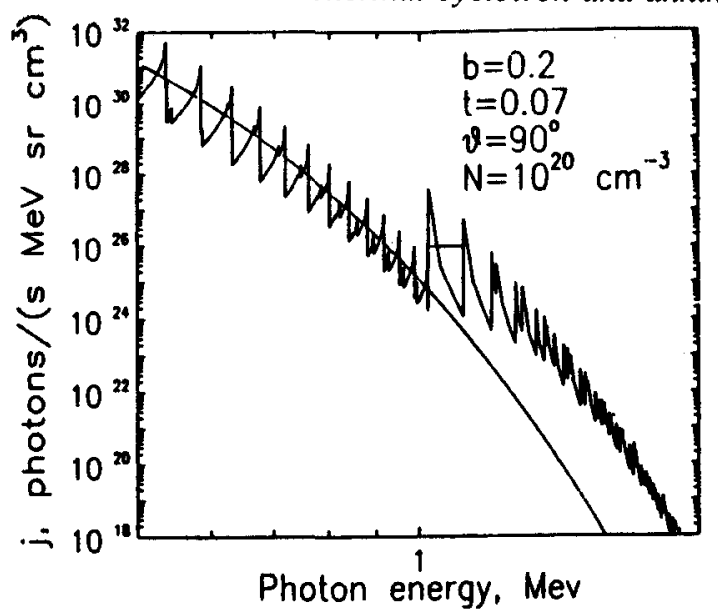

Fig. 30.3 Spectrum of differential emissivity $j$ near the annihilation jump for $T=0.07 m c^{2}, B=0.2 B_{c r}$ and $N=10^{20} \mathrm{~cm}^{-3}$. The bar between the first and the second annihilation peaks corresponds to the averaged value obtained from Eq. (7).

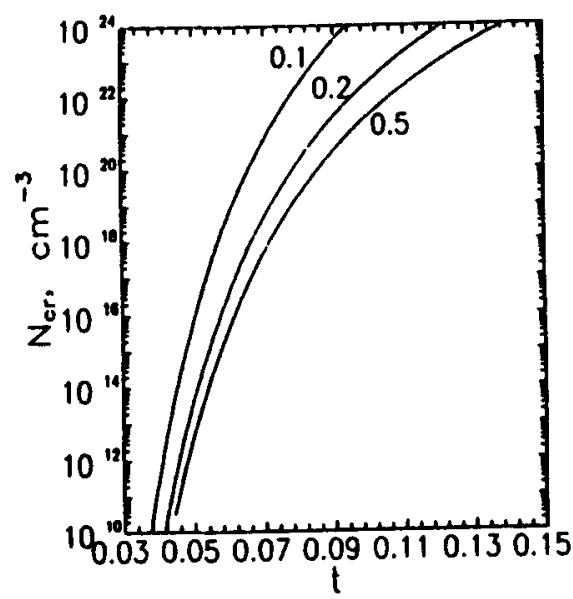

Fig. 30.4 Critical particle number density $N_{c r}$ as a function of $t=T / \mathrm{mc}^{2}$ for three values of $b=B / B_{c}$. (numbers near the curves).

\section{References}

Bezchastnov V.G. and Pavlov G.G., Astrophys.Sp.Sci. 178, 1, (1991)

Golenetskii S.V. et al., Nature 307, 41, (1984)

Harding A., Astrophys.J. 300, 167, (1986)

Harding A., Phys. Reports 206, 327, (1991)

Mazets E.P. et al., Astrophys.Sp.Sci. 84, 173, (1982) 